\title{
Metanephric Stromal Tumor
}

National Cancer Institute

\section{Source}

National Cancer Institute. Metanephric Stromal Tumor. NCI Thesaurus. Code C157749.

A rare benign renal neoplasm composed of moderately cellular spindle cells. It occurs mostly in infancy and childhood. 